\title{
Oxidative Stress Research on Huntington's Disease Neurons Using Genetically Encoded Biosensors
}

\section{Tuyana Malankhanova, Dayaana Vasileva, Elena Grigor'eva, Sergey Medvedev, Suren Zakian, and Anastasia Malakhova}

Federal Research Center Institute of Cytology and Genetics, the Siberian Branch of the Russian Academy of Sciences, Novosibirsk, Russia

\section{ORCID}

Tuyana Malankhanova; 0000-0002-5106-8465

Corresponding Author: Tuyana Malankhanova; email: tbmalankhanova@gmail.com

\section{Dates}

Published 13 January 2022

Publishing services provided by Knowledge E

(c) Tuyana Malankhanova et

al. This article is distributed

under the terms of the

which permits unrestricted use and redistribution provided that the original author and source are credited.

Selection and Peer-review under the responsibility of the 8th Scientific and Practical Conference Conference Committee.
Abstract. Many neurodegenerative diseases, including Huntington's disease (HD), are associated with oxidative stress in the neurons of the brain. Genetically encoded biosensorsare useful for studying these processesin vitro. Human cell cultures expressing the biosensors can serve as a cell model for developing and testing effective agents that reduce oxidative stress. In this work, transgenes encoding biosensors of glutathione oxidative potential(Grx1-roGFP2) with cytoplasmic and mitochondrial localization were introduced into human induced pluripotent stem cells of a healthy donor and an HD patient using CRISPR/Cas9-mediated genome editing. The cells were subsequently differentiated into medium spiny neurons of the striatum. The expression of the biosensors was detected in the iPSCs, neuronal precursors and mature neurons. The obtained cells could be used to study the redox potential of glutathione in HD neurons and to screen for new drug compounds aimed at reducing oxidative stress.

Keywords: genetically encoded biosensors, Huntington's disease, induced pluripotent stem cells, medium spiny neurons, oxidative stress, glutathione, Grx1-roGFP2

\section{Introduction}

Genetically encoded biosensors (GEBs) are perspective tools for real-time investigation of different processes in living cells. Biosensors based on fluorescent proteins are stable and do not affect the cells as compared to synthetic analogs. The protein expression is controlled, and it is possible to direct the sensor protein to different cell compartments such as mitochondria, cytoplasm, and ER. GEBs allow to investigate pathological processes, particularly, oxidative stress (OS). Glutathione is important antioxidant which recover disulfide bonds due to cysteine residue availability, so it shows redox status of cells. GEBs enable observing and measuring redox potential of glutathione by modified GFP (roGFP) which is an indicator of oxidized and reduced form ratio of glutathione. The redox protein glutaredoxin (Grx1) catalyzes the reaction of thiol-disulfide exchange between roGFP and glutathione [1]. 
OS is one of the key factors of Huntington's disease (HD) development. HD is a severe neurodegenerative autosomal dominant disease caused by CAG repeat expansion in the first exon of the HTTgene. It was shown that OS is caused by defect of mitochondrial functioning induced by mutant huntingtin (mHTT) [2]. Aggregatesofthemutant protein increase calciumioninflux,leadingto decrease in ATP production and apoptosis of striatum neurons. The striatumis an anatomical structure of telencephalon responsible for regulating muscle tone, functioning of internal organs, coordinating movements, cognitive processes and emotions. 95\% of the striatum cell population are medium spiny neurons (MSNs). MSNs are most affected neuronal type in HD.

In this work, we generated transgenic HD and healthy control iPSCs carrying Grx1roGFP2 biosensors with cytoplasmic and mitochondrial localization. We validated pluripotent state of the transgenic cells using standard tests. Moreover, we demonstrated that obtained cells can be efficiently differentiated into relevant medium spiny neurons. The resulting cells represent a valuable cell model to screen potential drugs reducing the OS level and to evaluate the redox potential of neurons at different stages of differentiation in various culture conditions.

\section{Material and Method}

\subsection{Materials}

iPSC line from a healthy donor K7 (https://hpscreg.eu/cell-line/ICGi022-A) and patientspecific iPSC line 47Q (https://hpscreg.eu/cell-line/ICGi007-A) with 47 CAG repeats in the first exon of HTTwere used [3].

\subsection{Methods}

\subsubsection{Cultivation of iPSCs}

iPSCs were cultured in KnockOut DMEM media with 15\% KoSR, 0.1 mM NEAA, 2mercapthoethanol, 1\% penicillin-streptomycin, GlutaMAX-I and $10 \mathrm{ng} / \mathrm{ml}$ bFGF onto mitotically inactivated mouse embryonic fibroblasts. iPSCs were dissociated with TrypLE (Life Technologies) and split at 1:10 twice a week. Cells were cultured at $37^{\circ} \mathrm{C}$ in $5 \%$ $\mathrm{CO} 2$

\subsubsection{Transfection and selection of recombinant cell clones}


TABLE 1: Plasmids used for iPSCs transfection.

$\begin{array}{lc}\text { Plasmids } & \text { Source } \\ \text { pX458-AAVS1 } & {[4]} \\ \text { pMito-Grx1-roGFP2-donor } & \text { (or [4] } \\ \text { pCyto-Grx1-roGFP2-donor) } & \\ \text { AAVS1-Neo-M2rtTA } & \text { Addgene \#60843, USA }\end{array}$

iPSCs were transfected using Neon Transfection System (Thermofisher Scientific). For the one transfection reaction, $5 \times 10^{5}$ cells and $5 \mu \mathrm{g}$ of total plasmid mix (Table 1) were used. Transfection parameters: $1100 \mathrm{~V}, 30 \mathrm{~ms}, 1$ pulse. At day 2 after transfection, the selection of recombinant clones was started using $300 \mathrm{ng} / \mathrm{ml}$ puromycin. The selection has been performed for 3 days. After selection when iPSC colonies reached optimal size, $100 \mathrm{ng} / \mathrm{ml}$ doxycycline was added to the culture medium. Green iPSCs colonies were picked manually and seeded into individual wells.

\subsubsection{Genome DNA extraction}

Genome DNA was extracted using Quick Extract (Epicentre) according to the manufacturer's protocol.

\subsubsection{PCR}

PCR was carried out in $20 \mu$ volume: $3 \mu$ of genome DNA, $0,5 \mu$ each of $10 \mu \mathrm{M}$ primers (Table 2), $10 \mu$ l BioMaster HS-Taq PCR-Color (2x) (Biolabmix), $6 \mu$ nuclease free deionized water. Reactions were run in $\mathrm{C} 1000$ Touch Thermal Cycler (Bio-Rad) using next program: $95^{\circ} \mathrm{C}, 5 \mathrm{~min} ; 35$ cycles: $95^{\circ} \mathrm{C}-30 \mathrm{~s}, 62^{\circ} \mathrm{C}-30 \mathrm{~s}, 72^{\circ} \mathrm{C}-30 \mathrm{~s} ; 72^{\circ} \mathrm{C}, 5 \mathrm{~min}$.

\subsubsection{Immunofluorescence staining}

iPSCs were fixed in $4 \%$ paraformaldehyde for $10 \mathrm{~min}$ at room temperature (RT), permeabilized in $0.5 \%$ Triton-X100 for 30 min at RT, then incubated with Blocking Buffer (BSA 1\% in PBS) for 30 min at RT. Primary antibodies were incubated overnight at $4{ }^{\circ} \mathrm{C}$ (Table 3). Secondary antibodies were added $1.5-2 \mathrm{~h}$ at RT (Table 3). All antibodies were diluted in PBS with 1\% BSA. Nuclei were counterstained with DAPI. Micrographs were taken using a Nikon eclipse Ti-E microscope and NIS Elements software. 
TABLE 2: Primers.

\begin{tabular}{|c|c|c|c|}
\hline $\begin{array}{l}\text { Pairs } \\
\text { primers }\end{array}$ & $\begin{array}{l}\text { Product } \\
\text { length }\end{array}$ & Sequence, $5^{\prime} \rightarrow>3^{\prime}$ & Comment \\
\hline $\begin{array}{l}\text { Puro-In-R HA- } \\
\text { L-OUT }\end{array}$ & 1062 & $\begin{array}{l}\text { AGGCGCACGTGGGCTTGTAC } \\
\text { CCGGACCACTTTGAGCTCTAC }\end{array}$ & $\begin{array}{l}\text { Biosensor } \\
\text { detection in } \\
\text { AAVS1 allele }\end{array}$ \\
\hline $\begin{array}{l}\text { Neo-In-R HA-L- } \\
\text { OUT }\end{array}$ & 1042 & $\begin{array}{l}\text { AGAACCTGCGTGCAATCCATC } \\
\text { CCGGACCACTTTGAGCTCTAC }\end{array}$ & $\begin{array}{l}\text { Transactivator } \\
\text { detection in } \\
\text { AAVS1 allele }\end{array}$ \\
\hline $\begin{array}{l}\text { Puro-In-R M13 } \\
\text { F }\end{array}$ & 1005 & $\begin{array}{l}\text { AGGCGCACGTGGGCTTGTAC } \\
\text { GTTGTAAAACGACGGCCAGTG }\end{array}$ & $\begin{array}{l}\text { Detection of } \\
\text { donor plasmid } \\
\text { DNA } \\
\text { biosensor with }\end{array}$ \\
\hline $\begin{array}{l}\text { Neo-In-R M13 } \\
\text { R }\end{array}$ & 1082 & $\begin{array}{l}\text { AGAACCTGCGTGCAATCCATC } \\
\text { AGCGGATAACAATTTCACACAGGA }\end{array}$ & $\begin{array}{l}\text { Detection of } \\
\text { donor plasmid } \\
\text { DNA with } \\
\text { transactivator }\end{array}$ \\
\hline
\end{tabular}

\subsubsection{In vitro spontaneous differentiation}

The iPSCs were increased in cultural dishes to $85-90 \%$ density, then cells disaggregated cells by adding $0,15 \%$ Collagenase IV (Thermo Fisher Scientific) for 40 min in $37^{\circ} \mathrm{C}$ with $5 \%$ CO2. Obtained cell embryoid bodies (EB) transferred in cultural cup coated 1\% agarose and cultivating in non-basic FGF medium for 2 weeks, then EB transferred in cultural dishes covered Matrigel-ESQ (Corning) and cultivating for 2 weeks.

\subsubsection{Differentiation of iPSCs into the MSNs}

Neuronal differentiation was performed as previously described protocol (Grigor'eva et al., 2020).

\section{Result and Discussion}

\subsection{Selection of recombinant cell clones}

For the transgene integration, the "safe harbor" locus AAVS1was chosen (Figure 1). The AAVS1 locus is a transcriptionally active region that provides transcriptional activity of the transgene [5]. Moreover, transgene integration into this locus does not disturb the expression of other genes. Two biosensors were used: Cyto-Grx1-roGFP2 and Mito-Grx1roGFP2, which are expressed in the cytoplasm or in mitochondria and allow evaluation of the redox potential in the neuron cytoplasm and mitochondria respectively. 
TABLE 3: Antibodies used for immunocytochemistry.

\begin{tabular}{|c|c|c|}
\hline Antibody & Company, Cat\# & Dilution \\
\hline \multicolumn{3}{|c|}{ Primary } \\
\hline Rabbit IgG anti-GABA & Sigma, ABN131 & $1: 500$ \\
\hline Mouse igG2a anti-TuJ1 & Covance, 801201 & 1:100 \\
\hline Rabbit IgG anti-NF200 & Sigma, N4142 & 1:1000 \\
\hline Chicken IgG anti-MAP2 & Abcam, ab5392 & 1:1000 \\
\hline Rabbit IgG anti-SOX2 & Cell Signaling, 3579 & $1: 500$ \\
\hline Mouse IgM anti-TRA-1-60 & Abcam, ab16288 & 1:200 \\
\hline Mouse IgG anti-NANOG & Abcam, ab62734 & 1:200 \\
\hline Mouse IgG anti-OCT4 & BD Transduction Lab, 611202 & $1: 50$ \\
\hline Mouse IgG anti- $\alpha$ SMA & DAKO, M0851 & $1: 100$ \\
\hline Mouse IgG3 anti-SSEA4 & Abcam, ab16287 & 1:200 \\
\hline Rabbit IgG anti-GATA62 & Cell Signaling, 5851 & $1: 50$ \\
\hline $\begin{array}{l}\text { Mouse igG1 anti- } \\
\text { Cytokeratin } 18\end{array}$ & Millipore, MAB3236 & $1: 200$ \\
\hline $\begin{array}{l}\text { Mouse igG1 anti-Collagen } \\
\text { IV }\end{array}$ & LifeSpanBiosciences, LS-C128254 & $1: 200$ \\
\hline Rabbit IgG anti-DARPP32 & Santa Cruz Biotechnology, H-62 & $1: 50$ \\
\hline \multicolumn{3}{|c|}{ Secondary } \\
\hline $\begin{array}{l}\text { Alexa Fluor } 488 \text { goat anti } \\
\text { rabbit } \lg G(\mathrm{H}+\mathrm{L})\end{array}$ & Thermo Fisher Scientific, A11078 & $1: 400$ \\
\hline $\begin{array}{l}\text { Alexa Fluor } 568 \text { goat anti } \\
\text { rabbit IgG }(H+L)\end{array}$ & Thermo Fisher Scientific, A-11011 & $1: 400$ \\
\hline $\begin{array}{l}\text { Alexa Fluor } 488 \text { goat anti } \\
\text { mouse } \lg G(\mathrm{H}+\mathrm{L})\end{array}$ & Thermo Fisher Scientific, A28175 & $1: 400$ \\
\hline $\begin{array}{l}\text { Alexa Fluor } 488 \text { goat anti } \\
\text { mouse IgG2a }\end{array}$ & Thermo Fisher Scientific, A-21131 & $1: 400$ \\
\hline $\begin{array}{l}\text { Alexa Fluor } 568 \text { goat anti } \\
\text { mouse } \operatorname{lgG} 2 a\end{array}$ & Thermo Fisher Scientific, A-21134 & $1: 400$ \\
\hline $\begin{array}{l}\text { Alexa Fluor } 488 \text { goat anti } \\
\text { mouse IgG1 }\end{array}$ & Thermo Fisher Scientific, A-21121 & $1: 400$ \\
\hline $\begin{array}{l}\text { Alexa Fluor } 488 \text { goat anti } \\
\text { mouse IgG3 }\end{array}$ & Thermo Fisher Scientific, A-21151 & $1: 400$ \\
\hline $\begin{array}{l}\text { Alexa Fluor } 568 \text { goat anti } \\
\text { mouse IgM }\end{array}$ & Thermo Fisher Scientific, A21043 & $1: 400$ \\
\hline $\begin{array}{l}\text { Alexa Fluor } 488 \text { rabbit anti } \\
\text { goat } \lg G(\mathrm{H}+\mathrm{L})\end{array}$ & Thermo Fisher Scientific, A27012 & $1: 400$ \\
\hline $\begin{array}{l}\text { Alexa Fluor } 568 \text { rabbit } \\
\text { anti goat } \operatorname{lgG}(\mathrm{H}+\mathrm{L})\end{array}$ & Thermo Fisher Scientific, A-11079 & $1: 400$ \\
\hline
\end{tabular}

Three plasmid constructs were used for transfection of the patient-specific line 47Q and healthy control line K7: pCyto-Grx1-roGFP2-donor/pMito-Grx1-roGFP2-donor containing a biosensor that expresses Grx1-roGFP2 in the cytoplasm or mitochondria, respectively, as well as a puromycin resistance gene; AAVS1-Neo-M2rtTA, encoding 


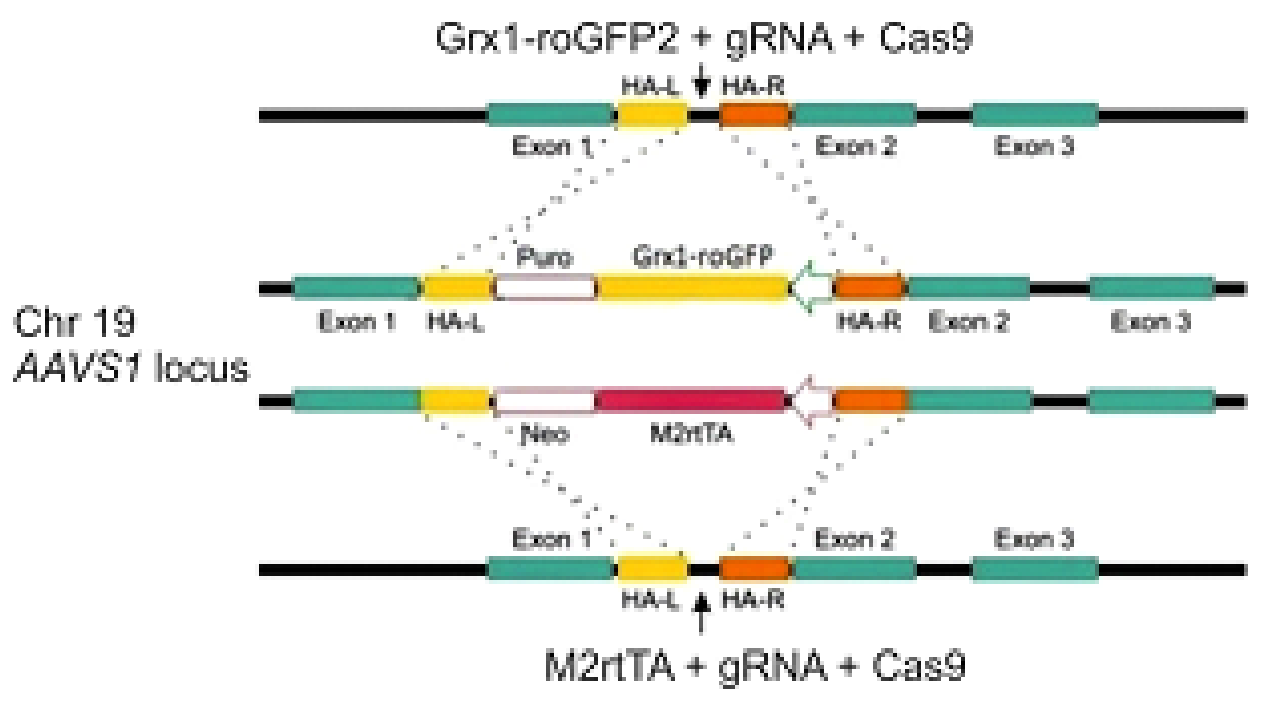

Figure 1: Scheme for integration of transgenes into the AAVS1 locus.

a tetracycline transactivator and a neomycin resistance gene; pX458-AAVS1 encoding elements of the CRISPR/Cas9 system with a guide RNA targeted to the AAVS1 locus.

As a result of the experiment, four 47Q clones with the Cyto-Grx1-roGFP2 and four 47Q clones with the Mito-Grx1-roGFP2 were obtained. We validated these clones for the presence of the transgene insertions by PCR using four pairs of primers: the first pair was used to detect the insertion of the biosensor into the AAVS1 locus, the second pair was to detect the insertion of the transactivator into the AAVS1 locus, the third and fourth pairs were to detect off-target insertions of the donor plasmids. Only cell clones without off-target insertions were used for further experiments. Figure 2 shows agarose gel electrophoresis of PCR screening of clones. PCR analysis of K7 cell clones revealed 9 clones of pMito-Grx1-roGFP2 and 14 clones of pCyto-Grx1-roGFP2 harbours both biosensor andtransactivator at the AAVS1 locus.

\subsection{Characterization of iPSCs with biosensors}

An important step of the study was confirmation that the cells have retained pluripotency aftertransgenesis and clonal isolation. The cells had a large nuclear-cytoplasmic ratio, had a high proliferative activity, the colonies demonstrated flat ESC-like morphology.

Immunofluorescence staining showed that all newly obtained lines of transgenic iPSCs expressed pluripotency markers: the main transcription factors OCT4, NANOG, SOX2, as well as surface antigens TRA-1-60, SSEA4 (Figure 3).

One of the main characteristics of iPSCs is the ability to differentiate into derivatives of three germ layers: ectoderm, mesoderm, and endoderm. To test this ability, we 


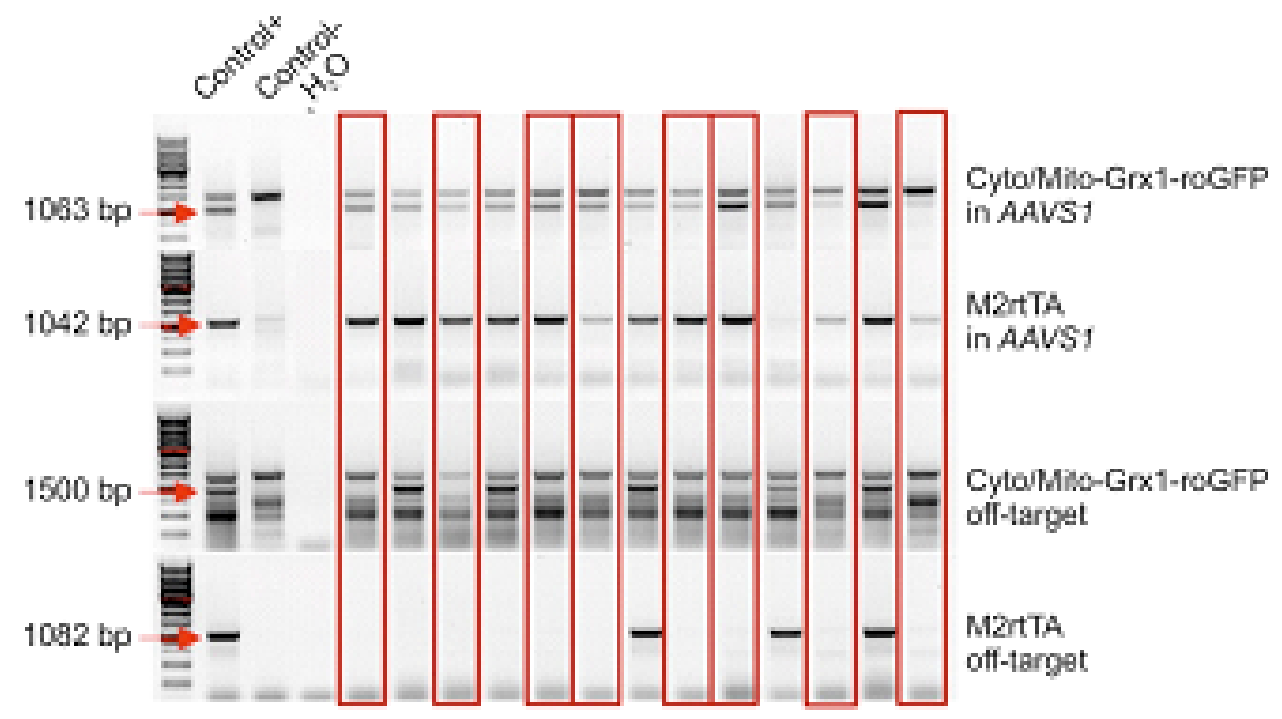

Figure 2: PCR screening of cell clones. Clones with insertions of transgenes into AAVS1 and without off-target insertions are in red frames.
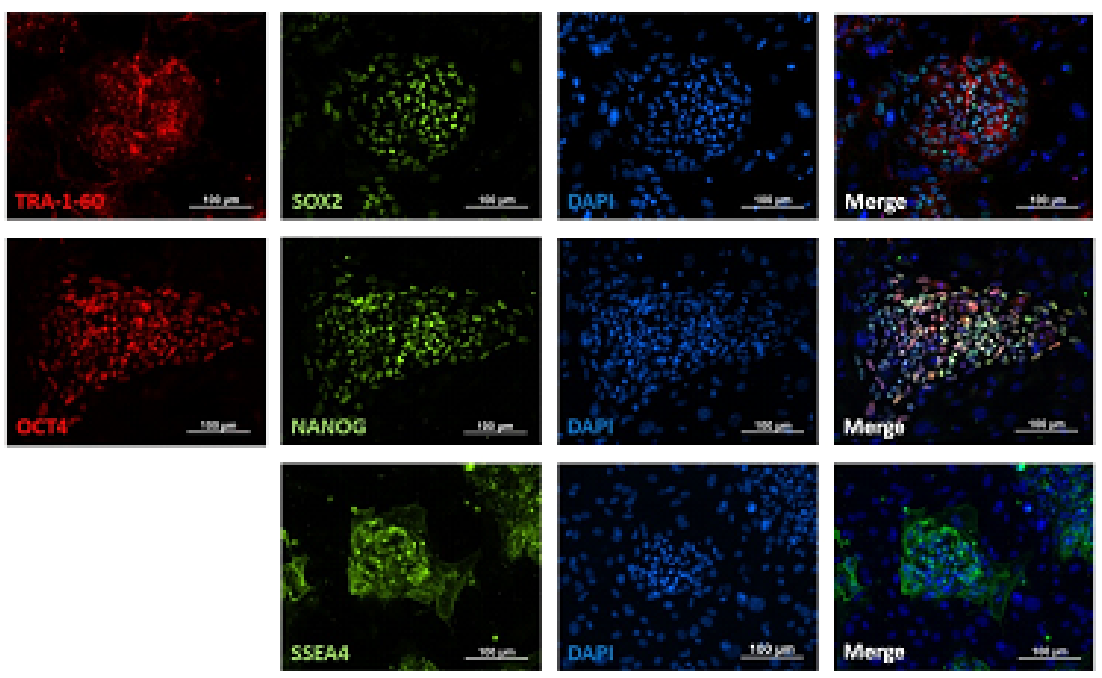

Figure 3: Immunofluorescence staining of iPSC clone 47Q-Cyto with antibodies against transcription factors: OCT4 (red signal), NANOG (green signal), SOX2 (green signal), TRA-1-60 (red signal) and SSEA4 (green signal). The nuclei are counterstained with DAPI (blue signal).

carried out spontaneous differentiation of iPSC lines (lines 47Q-pM2, 47Q-pC13) in vitro through the formation of embryoid bodies - spherical structures with intensively dividing and differentiating cells inside. Differentiation of embryoid bodies mimics the processesthat occur during the differentiation of the inner cell mass of the embryo in vivo. The structures obtained after 2 weeks of cultivation in suspension were spread on a matrix, after 9 days the cells were fixed on culture plates and immunofluorescence analysis was performed.

Spread embryoid bodies of all clones obtained in the work were stained with antibodies against markers of ectoderm - proteins of the cytoskeleton of neurons TUJ1 (or 

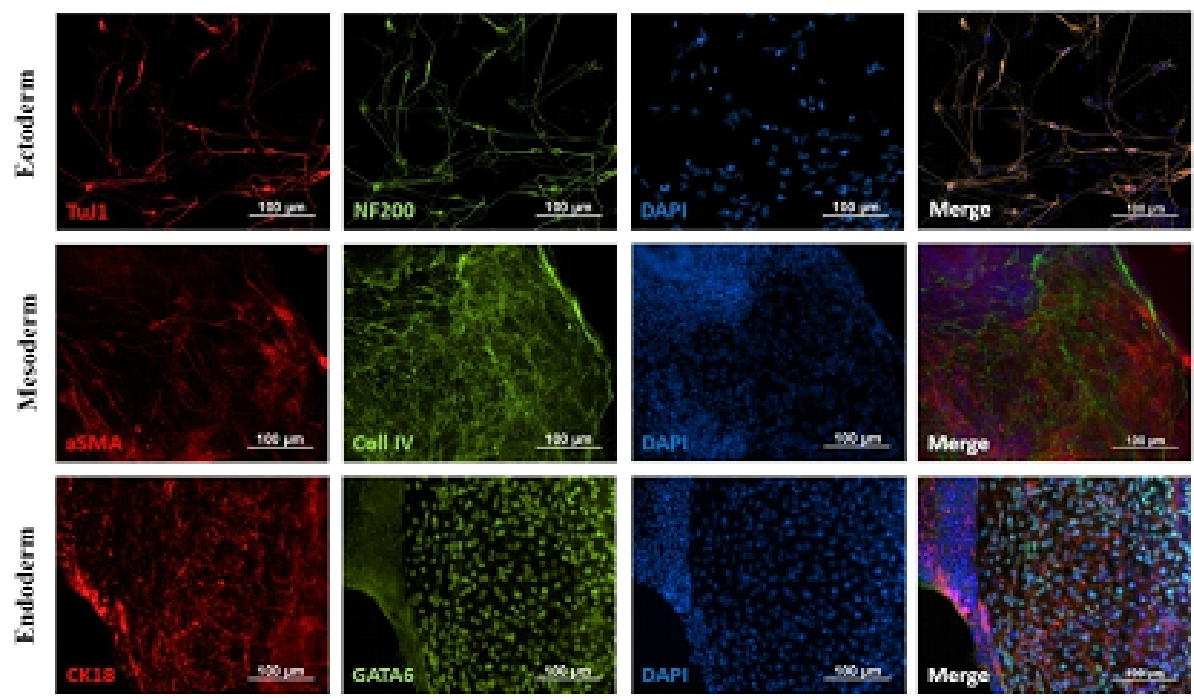

Figure 4: Immunofluorescence staining of spread embryoid bodies obtained after 47Q-Mito spontaneous differentiation.: $\alpha$-SMA (red signal), COLL IV (green signal); CK18 (red signal), GATA6 (green signal); TUJ1 (red signal), NF200 (green signal). The nuclei are counterstained with DAPI (blue signal).

BIII-tubulin) and NF200; mesoderm - isoforms of smooth muscle cell actin $\alpha$-SMA and the main component of the connective tissue collagen IV (COLL IV), and endoderm - transcription factors required for endodermal differentiation of organ tissues GATA6 and CYTOKERATIN 18 (CK18) (Figure 4).

Thus, the expression of the main markers of pluripotency of iPSC lines was demonstrated, as well as the ability to differentiate into derivatives of three germ layers. These methods confirm the transgenic iPSCs saved pluripotent properties after genome modifications.

\subsection{Differentiation of iPSC into the MSNs}

After characterization of the obtained transgenic iPSC lines, we carried out directed differentiation into MSNs, a type of neurons that are most affected in HD. We performed differentiation using a previously developed protocol (Grigor'eva et al., 2020).

For differentiation, iPSCs were seeded onto a Matrigel-ESQ matrix, grown to 80$90 \%$ confluency, and differentiated to the neuroectodermal direction. At the first stage, dual SMAD inhibition occurred by the addition of small molecules such as LDN193189 and SB431542 to the growth medium. Addition of the Purmorphamine to the medium ensured differentiation into neurons. Dorsomorphin facilitated further differentiation of iPSCs to the neural direction.

After 12 day of differentiation, the cells were detached using the Accutase enzyme and plated in a 1:2 ratio onto aMatrigel-ESQ matrix coated culture surface in NeuroB medium. 

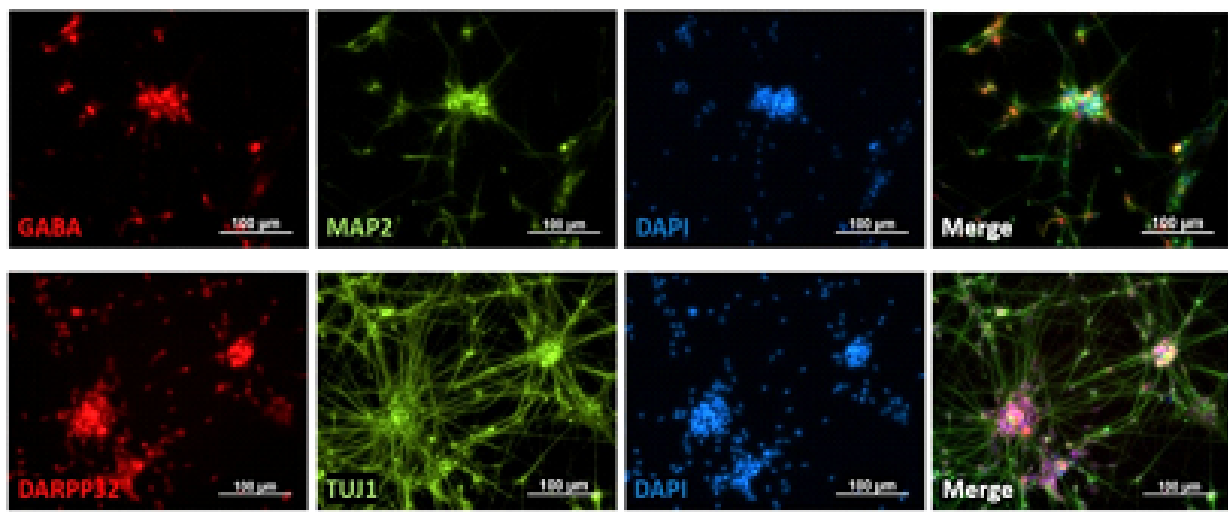

Figure 5: Immunofluorescence staining of terminally differentiated MSNs (clone 47Q-Cyto) at 13th day of the terminal differentiation.

The differentiation medium contained the neurotrophic factor BDNF that stimulates and supports the development of neurons, increasing their survival and number, as well as differentiation, which led to intensive cell proliferation. The presence of ascorbic acid during differentiation ensured the maturation of neurons and exerted antioxidant and neuroprotective effects [6].

After 2-3 passages, MSN progenitors were seeded for the terminal stage of differentiation. Cells were seeded at a density $1-1.5 \times 10^{4}$ cells $/ \mathrm{cm}^{2}$ and cultured in medium containing BDNF, ascorbic acid, CNTF, compound E, and CAMP.

Immunofluorescence analysis on the 13th day of the terminal differentiation showed that cells expressed the main neuronal markers - MAP2 and TUJ1, as well as markers of MSNs - DARPP32 and GABA (Figure 5).

\subsection{Confirmation of biosensors expression}

The activation of GEB expression depends on the presence of doxycycline in the culture medium. Doxycycline binds to the transactivator, and this complex triggers the expression of the biosensor. Therefore, to check the functionality of the biosensor, doxycycline was added to the cells at different stages of differentiation - iPSCs, MSN progenitors and MSNs.

We evaluated the expression of the Grx1-roGFP2 biosensor in the 47Q-Cyto and 47Q-Mito clones (Figure 6). The cells with the cytoplasmic biosensor had scattered fluorescence light due to the fact that the biosensor is distributed throughout the cell in the cytoplasm. Cells with the mitochondrial biosensor had bright and clear fluorescnce due to the dense localization of the biosensor protein in the mitochondria. 

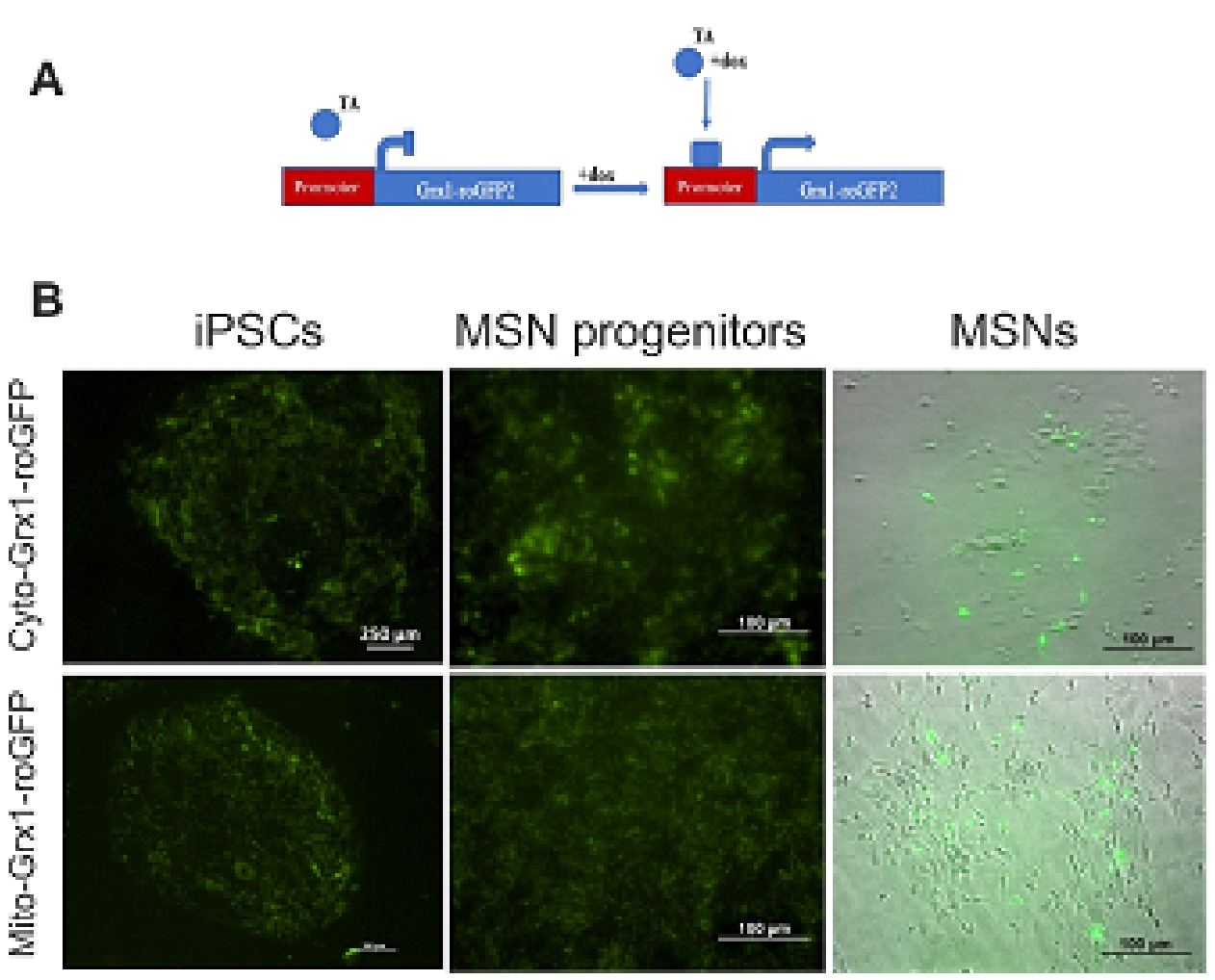

Figure 6: Biosensor expression. A - Scheme of biosensor expression activation. B - Fluorescence of the biosensor at different stages of differentiation.

\section{Conclusion}

Thus, in this work, anunique HD and control iPSC-based platform was created for studying oxidative stress in HD pathogenesis. It was shown that the clones contained two transgenes in the AAVS1 locus: the genetically encoded biosensor (cytoplasmic or mitochondrial) and the tetracycline transactivator.

After transgenesis, these clones retained their pluripotent state that was shown by immunofluorescence staining for the main markers of pluripotent stem cells and spontaneous differentiation in vitro. Further, transgenic clones were differentiated into a special type of cells that degenerates in HD - medium spiny neurons.

This cell platform enables to investigate OS at various stages of the disease progression both at the cellular and molecular levels. Due to the fact that iPSCs proliferate indefinitely, as well as the progenitors of MSNs are capable of long-time proliferation, it becomes possible to build up a large number of differentiated neuronsthat can then be used for screening potential drugs aimed at reducing the total OS level in cells. 


\section{Acknowledgments}

This work was supported by the Russian Science Foundation project No 16-15-10128.

\section{References}

[1] Bilan D.S., Lukyanov S.A., Belousov V.V.Genetically Encoded Fluorescent Redox Sensors]. Bioorganic chemistry. 201541 (3) 259-274

[2] Lee JM, Ramos EM, Lee JH et al. CAG repeat expansion in Huntington disease determines age at onset in a fully dominant fashion. Neurology. 2012 78(10):690695. doi: 10.1212/WNL.0b013e318249f683.

[3] Grigor'eva, Elena \& Malankhanova, Tuyana \& Surumbayeva, Aizhan et al. Generation of induced pluripotent stem cell line, ICGi007-A, by reprogramming peripheral blood mononuclear cells from a patient with Huntington's disease. Stem Cell Research. 2019. 34. 101382-101382. 10.1016/j.scr.2018.101382.

[4] Ustyantseva El, Medvedev SP, Vetchinova AS, Minina JM, Illarioshkin SN, Zakian SM. A Platform for Studying Neurodegeneration Mechanisms Using Genetically Encoded Biosensors. Biochemistry (Mosc). 2019;84(3): 299-309.

[5] Pellenz S, Phelps M, Tang W, Hovde BT, Sinit RB, Fu W, Li H, Chen E, Monnat RJ Jr. New Human Chromosomal Sites with "Safe Harbor" Potential for Targeted Transgene Insertion. Hum Gene Ther. 2019 Jul;30(7):814-828. doi: 10.1089/hum.2018.169.

[6] Grigor'eva EV, Malankhanova TB, Surumbayeva A et al. Generation of GABAergic striatal neurons by a novel iPSC differentiation protocol enabling scalability and cryopreservation of progenitor cells. Cytotechnology. 2020 72(5):649-663. doi: 10.1007/s10616-020-00406-7 